\title{
Clonal selection in the germinal centre by regulated proliferation and hypermutation
}

\author{
Alexander D. Gitlin ${ }^{1}$, Ziv Shulman ${ }^{1} \&$ Michel C. Nussenzweig ${ }^{1,2}$
}

During immune responses, B lymphocytes clonally expand and undergo secondary diversification of their immunoglobulin genes in germinal centres $(\mathrm{GCs})^{1-4}$. High-affinity $B$ cells are expanded through iterative interzonal cycles of division and hypermutation in the GC dark zone followed by migration to the GC light zone, where they are selected on the basis of affinity to return to the dark zone $e^{5-10}$. Here we combine a transgenic strategy to measure cell division and a photoactivatable fluorescent reporter to examine whether the extent of clonal expansion and hypermutation are regulated during interzonal GC cycles. We find that both cell division and hypermutation are directly proportional to the amount of antigen captured and presented by GC B cells to follicular helper T cells in the light zone. Our data explain how GC B cells with the highest affinity for antigen are selectively expanded and diversified.

Clonal expansion is an essential feature of the immune response. $\mathrm{B}$ lymphocytes bearing antigen-specific immunoglobulins undergo this process in the germinal centre, a specialized microanatomical compartment where B cells also diversify their immunoglobulin genes through somatic hypermutation ${ }^{1-4}$. GC B cells expressing mutated surface immunoglobulins with the highest affinity are then positively selected by iterative cycles of cell division, somatic hypermutation and selection ${ }^{5-10}$, endowing the host with high-affinity humoral immunity ${ }^{4}$.

GC B cells divide and mutate in the dark zone (DZ), and then migrate to the light zone (LZ) where they capture antigen through surface immunoglobulin and present it as peptide bound to major histocompatibility complex class II (pMHCII) to cognate follicular helper T cells $\left(\mathrm{T}_{\mathrm{FH}}\right)^{4,10-12}$. Migration between the two zones is mediated by the chemokine receptors CXCR4 and CXCR5, with 50\% of DZ cells migrating to the LZ, and $10 \%$ returning to the $\mathrm{DZ}$ from the $\mathrm{LZ}$ within $6 \mathrm{~h}^{5,10,13}$. Moreover, $\mathrm{B}$ cells in the two GC zones alternate between distinct genetic programs reflecting cell division in the $\mathrm{DZ}$ and selection in the LZ, but do so independently of local cues received in the two zones ${ }^{10,14}$. However, the precise mechanism by which the highest affinity cells are selected, and whether cell divisions and immunoglobulin mutations in the DZ are regulated, remains unknown ${ }^{14}$.

To determine whether the amount of antigen internalized by GC B cells governs the extent of clonal expansion, we titrated the amount of antigen delivered to GC B cells using antibodies that target DEC205, an endocytic receptor that carries antigen to intracellular MHCII-containing compartments ${ }^{10,15-18}$. GC responses were initiated by priming mice with ovalbumin (OVA), followed by boosting with OVA coupled to the hapten 4-hydroxy-3-nitrophenylacetyl (NP-OVA) ${ }^{9}$. Antigen-specific B-cell responses were tracked by adoptive transfer of B1- $8^{\text {hi }}$ immunoglobulin heavy chain knock-in B cells, which are specific for NP when they express immunoglobulin lambda $(\operatorname{Ig} \lambda)$ light chains ${ }^{19}$. To measure the relative expansion of B cells receiving graded amounts of antigen, GCs were induced in mice that received a mixture of $\mathrm{B} 1-8^{\text {hi }} \mathrm{DEC} 205^{+/+}$ and $\mathrm{B} 1-8^{\mathrm{hi}} \mathrm{DEC} 205^{-/-} \mathrm{B}$ cells at a 5:95 ratio. Graded doses of antigen were delivered to DEC205 ${ }^{+/+}$GC B cells using the chimaeric DEC205 antibody fused to cognate antigen, OVA $(\alpha \mathrm{DEC}-\mathrm{OVA} \text {, Fig. 1a })^{20}$. Whereas control injections with PBS had no effect, injection with $10 \mu \mathrm{g}$ of $\alpha \mathrm{DEC}-$ OVA resulted in selective expansion of the $\mathrm{B} 1-8^{\text {hi }}$ DEC205 ${ }^{+/+}$GC B cells
(Fig. 1b, $c$ and Extended Data Fig. 1). Decreasing the dose of antigen delivered, by mixing $\alpha$ DEC-OVA with a chimaeric DEC205 antibody carrying the control irrelevant antigen Plasmodium falciparum circumsporozoite protein $(\alpha \mathrm{DEC}-\mathrm{CS})$, resulted in decreased expansion of

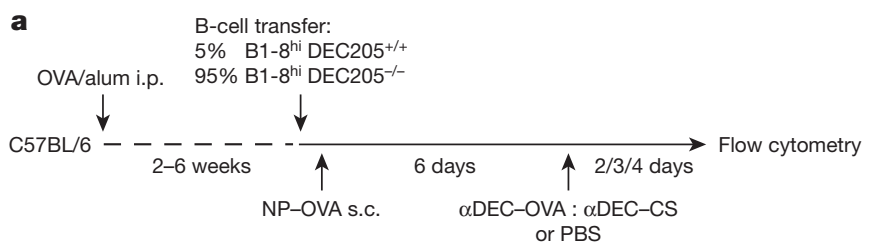

b

48 hours, gated on $\mathrm{B} 1-8^{\text {hi }} \mathrm{GC}$ B cells:
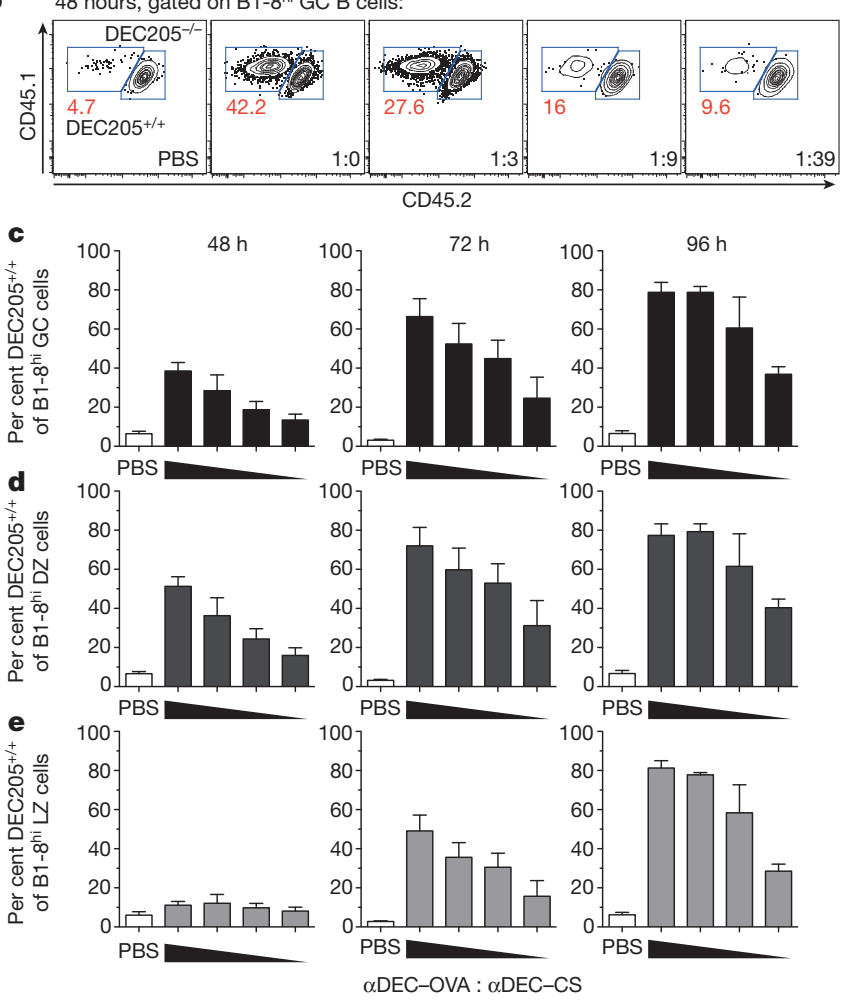

Figure $1 \mid$ The amount of antigen captured and presented by GC B cells regulates their expansion. a, Protocol for $\mathbf{b}-\mathbf{e}$. i.p., intraperitoneally; s.c.,

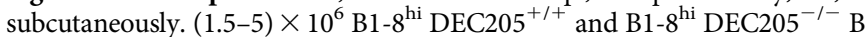
cells $\left(\approx(1.5-5) \times 10^{5} \mathrm{Ig} \lambda^{+}, \mathrm{NP}\right.$-specific B cells $)$ at a 5:95 ratio were transferred into OVA-primed wild-type mice, which were boosted with NP-OVA. After 6 days, mice were injected with PBS or $\alpha$ DEC-OVA mixed with $\alpha$ DEC-CS at ratios of 1:0, 1:3, 1:9, or 1:39. Lymph nodes were analysed 2, 3 and 4 days after injection. b, Proportion of B1-8 $8^{\text {hi }}$ DEC20 $5^{+/+}$and B1 $-8^{\text {hi }}$ DEC205 $5^{-/-}$GC $\mathrm{B}$ cells $48 \mathrm{~h}$ after treatment. c-e, Mean fraction of DEC205 $5^{+/+} \mathrm{B}$ cells among B1- $8^{\text {hi }} \mathrm{GC}(\mathbf{c}), \mathrm{DZ}\left(\mathrm{CD} 86^{-} \mathrm{CXCR}^{+}, \mathbf{d}\right)$, and LZ $\left(\mathrm{CD} 86^{+} \mathrm{CXCR}^{-}, \mathbf{e}\right)$ cells. Error bars, s.e.m. Data represent 2-3 independent experiments at each time point with a total of 4-6 mice per condition for all time points. 
B1-8 ${ }^{\text {hi }}$ DEC205 $5^{+/+}$GC B cells that was proportional to the dose of $\alpha \mathrm{DEC}-\mathrm{OVA}$ (Fig. 1b, c). Consistent with the idea that pMHCII-mediated selection occurs in the $\mathrm{LZ}$ and cell division in the $\mathrm{DZ}^{4,10}$, selective dosedependent expansion of B1-8 ${ }^{\text {hi }}$ DEC205 ${ }^{+/+}$GC B cells was already evident at $48 \mathrm{~h}$ in the DZ but only later in the LZ (Fig. 1d, e). In contrast, the $\mathrm{B} 1-8^{\text {hi }}$ DEC205 $5^{-/-}$GC B-cell population contracted in proportion to the amount of antigen delivered to the B1- $8^{\text {hi }}$ DEC20 $5^{+/+}$GC B-cell population (Fig. $1 \mathrm{~b}$ and Extended Data Fig. 1c). Thus, increasing the amount of cognate antigen presented by a subset of GC B cells to $\mathrm{T}_{\mathrm{FH}}$ cells leads to their proportional and selective expansion at the expense of GC B cells that present less antigen.

To examine the mechanism by which increased T-cell help leads to selective GC B-cell expansion, we sought to measure cell division in the GC. Traditional dye-based methods to monitor cell division are unsuitable in this context because $\mathrm{B}$ cells divide extensively and lose most of the dye before entering the GC. To circumvent this problem, we combined transgenes encoding the tetracycline transactivator (tTA) protein expressed under the Vav promoter and a histone H2B-mCherry fusion protein driven by a doxycycline (DOX)-regulated promoter (tTAH2B-mCh, Extended Data Fig. 2a) ${ }^{21,22}$. Under steady state conditions, tTA is expressed in haematopoietic cells and induces high levels of H2B-mCh expression (Extended Data Fig. 2b). Administration of DOX represses further $\mathrm{H} 2 \mathrm{~B}-\mathrm{mCh}$ synthesis, and as a result the $\mathrm{H} 2 \mathrm{~B}-\mathrm{mCh}$ dilutes in proportion to cell division (Extended Data Fig. $2 \mathrm{c})^{23}$.

To determine whether $\mathrm{TTA}-\mathrm{H} 2 \mathrm{~B}-\mathrm{mCh}$ can be used to track antigenspecific GC B-cell division in vivo, we repeated the prime-boost protocol described above using B1-8 ${ }^{\text {hi }}$ tTA-H2B-mCh B cells. As expected, non-proliferative follicular $\mathrm{B}$ cells did not dilute $\mathrm{H} 2 \mathrm{~B}-\mathrm{mCh}$ after DOX treatment (Fig. 2a). In contrast, after $36 \mathrm{~h}$, a spectrum of discrete peaks of $\mathrm{H} 2 \mathrm{~B}-\mathrm{mCh}$ expression corresponding to cell divisions became evident among GC B cells, and by $84 \mathrm{~h}, \mathrm{GC} B$ cells had completely diluted $\mathrm{H} 2 \mathrm{~B}-\mathrm{mCh}$ (Fig. 2a). Thus, tTA-H2B-mCh can be used to monitor cell division in the GC.

To determine whether the amount of antigen captured by GC B cells influences their degree of proliferation, we delivered additional antigen to GC B cells using $\alpha$ DEC-OVA. After $60 \mathrm{~h}$ on DOX, B1- $8{ }^{\text {hi }}$ DEC20 $55^{+/+}$ tTA-H2B-mCh GC B cells from mice treated with control $\alpha$ DEC-CS or PBS were nearly evenly distributed among $\mathrm{mCh}^{\mathrm{Med}}, \mathrm{mCh}^{\mathrm{Lo}}$ and $\mathrm{mCh}^{-}$ groups, representing cells that underwent progressively more cell division. In contrast, after $60 \mathrm{~h}$ on DOX, $\sim 50 \%$ of GC B cells targeted with $\alpha$ DEC-OVA became $\mathrm{mCh}^{-}$and $\sim 40 \%$ were $\mathrm{mCh}^{\text {Lo }}$ (Fig. $2 \mathrm{~b}, \mathrm{c}$ ). Therefore, increased antigen capture and presentation leads to increased rates of cell division by GC B cells.

Concomitant with the increased rate of cell division, there was a change in the zonal distribution of GC B cells. Whereas control cells equilibrated between DZ and LZ at a 2:1 ratio on average, $\alpha$ DEC-OVA-targeted GC $B$ cells were found almost exclusively in the DZ ( $~ 90 \%$, Fig. $2 d)$.

To examine cell cycle distribution during selection, we labelled GC B cells with an intravenous pulse of the nucleoside analogs 5-ethynyl2 '-deoxyuridine (EdU) followed $1 \mathrm{~h}$ later by 5 -bromo-2' -deoxyuridine (BrdU), allowing separation of GC B cells into the earliest $\left(\mathrm{EdU}^{-} \mathrm{BrdU}^{+}\right)$, $\mathrm{mid} / \mathrm{late}\left(\mathrm{EdU}^{+} \mathrm{BrdU}^{+}\right)$and post- $\left(\mathrm{EdU}^{+} \mathrm{BrdU}^{-}\right) \mathrm{S}$ phase periods of the cell cycle (Fig. 3a and Extended Data Fig. 3). Control GC B cells in early and mid/late-S phases were found in both zones; post-S phase cells were primarily in the DZ (Fig. 3b). This supports the idea that GC $B$ cells can initiate $S$ phase in the $L Z$ and then migrate to the DZ to divide $^{10}$. Moreover, the fact that early $S$ phase cells are distributed at a 2:1 DZ:LZ ratio suggests that, once in the DZ, GC B cells can initiate two cell divisions on average. In contrast, inducing selection by injecting $\alpha$ DEC-OVA shifted early $S$ phase cells among selected GC B cells to the DZ $(\sim 86 \%)$, whereas slightly fewer early $S$ phase cells were in the DZ (59\%) among non-selected cells (Fig. 3b). We conclude that increasing the amount of antigen captured and presented to $\mathrm{T}_{\mathrm{FH}}$ cells increases the proportion of cells initiating $S$ phase in the DZ. These findings indicate that GC B cells presenting the highest levels of antigen initiate additional cell divisions in the DZ before returning to the LZ.
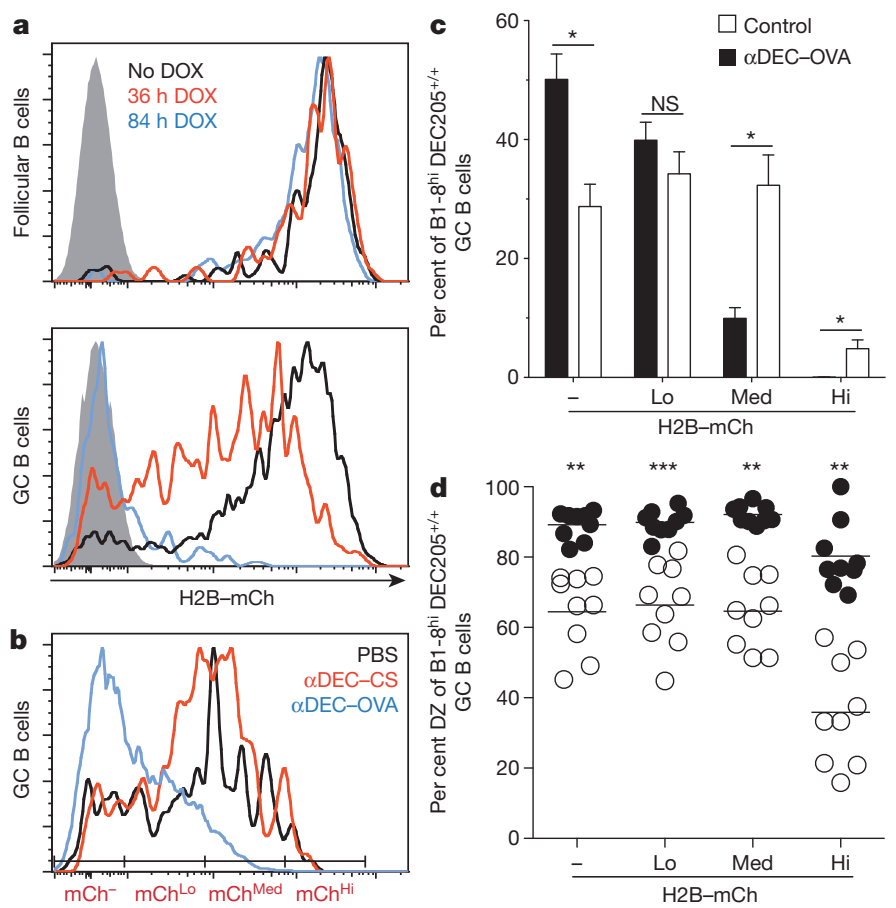

Figure $2 \mid$ T-cell help regulates the number of GC B-cell divisions.

a, H2B-mCh fluorescence among B1-8 ${ }^{\text {hi }}$ tTA-H2B-mCh B cells within the follicular (upper) or GC (lower) compartments of untreated mice (black) or after 36 (red) or 84 (blue) hours on DOX. Solid grey represents non-fluorescent cells. b, H2B-mCh fluorescence among B1- ${ }^{\text {hi }}$ tTA-H2B-mCh GC B cells in mice that received a 5:95 mixture of $\mathrm{B} 1-8^{\text {hi }}$ tTA-H2B-mCh and B1- $8^{\text {hi }}$ DEC205 ${ }^{-1-}$ B cells and were treated with PBS, $\alpha$ DEC-CS or $\alpha$ DEC-OVA for $72 \mathrm{~h}$ and administered DOX for $60 \mathrm{~h}$ before analysis. c, Mean fraction of B1-8 ${ }^{\text {hi }}$ tTA-H2B-mCh GC B cells treated as in b. Error bars, s.e.m. d, Per cent DZ cells among B1-8 ${ }^{\text {hi }}$ tTA-H2B-mCh GC B cells after treatment with PBS or $\alpha$ DEC-CS (control) or $\alpha$ DEC-OVA for $48 \mathrm{~h}$ and DOX for $36 \mathrm{~h}$. Each symbol represents one mouse and lines represent mean values. Data represent 3-4 independent experiments for all time points with $2-3$ mice for each condition per experiment. $* P<0.005$; $* * P<0.001$; ${ }^{*} * P<0.0001$, two-tailed

Mann-Whitney test.

We used photoactivation to test whether selected $B$ cells presenting higher levels of antigen reside longer in the $\mathrm{DZ}^{10}$. Primed mice received a mixture of B1-8 ${ }^{\text {hi }}$ DEC20 $5^{+/+}$B cells expressing a photoactivatable green fluorescent protein $\left(\mathrm{B} 1-8^{\text {hi }} \mathrm{PAGFP}^{+}\right.$) and $\mathrm{B} 1-8^{\mathrm{hi}} \mathrm{DEC} 205^{-1-} \mathrm{B}$ cells. On day 6 of the GC response, we induced selection by injecting $\alpha \mathrm{DEC}-\mathrm{OVA}$. DZ cells were photoactivated and lymph nodes were processed for flow cytometry either 0 or $6 \mathrm{~h}$ after photoactivation (Fig. 3c and Extended Data Fig. 4). Under control conditions, $57 \%$ of photoactivated GC B cells migrated to the LZ after $6 \mathrm{~h}^{10}$. In contrast, B1-8 ${ }^{\text {hi }}$ $\mathrm{PAGFP}^{+}$cells undergoing selective expansion migrated from the DZ to the LZ at half this rate (Fig. $3 \mathrm{~d}, \mathrm{e}$ ). Thus, GC B cells undergoing selective expansion as a result of increased antigen presentation reside longer in the $\mathrm{DZ}$, allowing them to undergo additional cell divisions before returning to the $\mathrm{LZ}$. We conclude that $\mathrm{T}_{\mathrm{FH}}$ cells regulate the number of cell cycles a GC B cell initiates during each passage through the DZ, and that they do so in direct proportion to the amount of antigen captured and presented.

To determine whether variable numbers of cell divisions in the $\mathrm{DZ}$ are associated with selection during a polyclonal immune response, we immunized non-immunoglobulin transgenic, tTA-H2B-mCh mice with NP-OVA. Mice were given DOX at day 12.5 after immunization, and $36 \mathrm{~h}$ later $\mathrm{mCh}^{\mathrm{Hi}}$ and $\mathrm{mCh}^{\mathrm{Lo}} \mathrm{GC} \mathrm{B}$ cells, representing lower and higher rates of proliferation, respectively, were isolated by cell sorting (Fig. 4a, b). $V_{H} 186.2$ family genes were then analysed for the characteristic high affinity anti-NP W33L mutation ${ }^{24}$. We found that $35.9 \%$, of the highly divided GC B cells were $\mathrm{W} 33 \mathrm{~L}^{+}$; in contrast, only $6 \%$ of the cells that underwent 

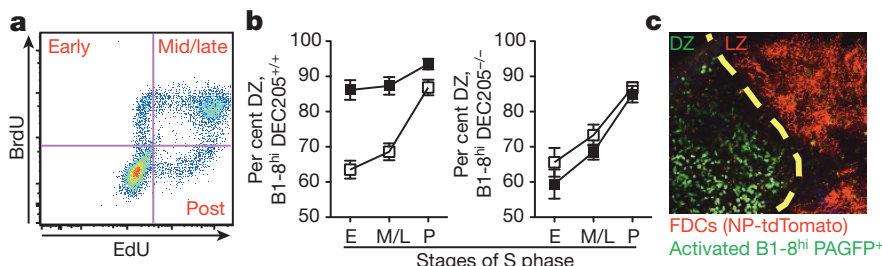

d
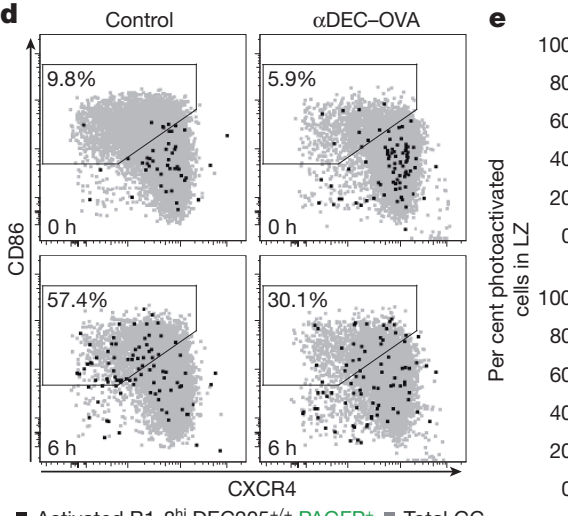

Figure 3 Selective expansion involves increased $S$ phase initiation in the $\mathrm{DZ}$ and longer $\mathrm{DZ}$ residence time. a, EdU and BrdU incorporation among GC B cells. OVA-primed mice received $5 \times 10^{6} \mathrm{~B} 1-8^{\mathrm{hi}} \mathrm{DEC} 205^{+/+}$and B1- $8^{\mathrm{hi}}$ DEC205 $5^{-1-}$ B cells at a 15:85 or 50:50 ratio. On day 6 after boosting with NP-OVA, mice receiving the 15:85 or the 50:50 B-cell transfer were injected with $\alpha$ DEC-OVA or PBS, respectively. Two days later, mice received EdU and $1 \mathrm{~h}$ later BrdU and were analysed after $30 \mathrm{~min}$ (detailed in Extended Data Fig. 3a). b, Per cent DZ cells among early (E), mid/late (M/L), and post- (P) S phase cells. Black squares denote $\alpha \mathrm{DEC}-\mathrm{OVA}$ treatment; white squares denote PBS control. Data represent two independent experiments with 6 or 7 mice per condition in total. Squares indicate mean values; error bars, s.e.m. c, d, OVA-primed mice received $5 \times 10^{6} \mathrm{~B}^{-}-8^{\text {hi }} \mathrm{PAGFP}^{+}$and $\mathrm{B} 1-8^{\mathrm{hi}}$ DEC205 ${ }^{-l-}$ B cells at a $15: 85$ ratio or $5 \times 10^{6}{\mathrm{~B} 1-8^{\text {hi }}}^{\text {PAGFP }}{ }^{+}$B cells alone and were boosted with NP-OVA. For mice receiving mixed B-cell transfers, $\alpha \mathrm{DEC}-\mathrm{OVA}$ was injected on day 6 . On day 8, DZ cells were photoactivated (c) and GC B cells were analysed by flow cytometry (detailed in Extended Data Fig. 4a) (d). e, Per cent LZ cells among photoactivated B1- $8{ }^{\text {hi }}$ PAGFP $^{+}$GC $B$ cells at 0 or $6 \mathrm{~h}$ after DZ photoactivation. Each symbol represents one mouse. Data represent multiple independent experiments. ${ }^{*} P=0.0022$, two-tailed Mann-Whitney test.

the fewest divisions were W33L $\mathrm{L}^{+}(P<0.0001$, Fig. 4b). Thus, selection of high-affinity GC B cells in a polyclonal response is associated with increased rates of proliferation. Moreover, because each division can introduce mutations that increase or decrease affinity, even high-affinity clones will rapidly partition into subclones that undergo different rates of proliferation, death or differentiation. This is probably reflected in the observation that $\mathrm{mCh}{ }^{\mathrm{Lo}}$ cells are not all $\mathrm{W} 33 \mathrm{~L}^{+}$, and that $\mathrm{W} 33 \mathrm{~L}^{+}$ cells are not exclusively in the $\mathrm{mCh}^{\text {Lo }}$ fraction.

Each round of cell division in the GC is predicted to produce 1 somatic mutation per $10^{3}$ base pairs in immunoglobulin genes ${ }^{8}$. The finding that $\mathrm{T}_{\mathrm{FH}}$ cells control the number of B-cell divisions per GC cycle indicates that they may also regulate hypermutation. To determine whether there is a correlation between selection, proliferation and somatic hypermutation in the GC, we immunized tTA-H2B-mCh mice with NP-OVA, purified $\mathrm{mCh}^{\mathrm{Hi}}$ and $\mathrm{mCh}{ }^{\mathrm{Lo}} \mathrm{GC} \mathrm{B}$ cells by cell sorting and analysed the intron downstream of $J_{H} 4$ for somatic mutations, because this region is targeted for somatic hypermutation but is not subject to selection ${ }^{25}$. GC B cells that had diluted $\mathrm{H} 2 \mathrm{~B}-\mathrm{mCh}$ by undergoing a greater number of divisions were significantly more mutated than those that had undergone fewer divisions $(P<0.0001$, Fig. $4 \mathrm{c})$.

High levels of mutation are required to produce broadly neutralizing antibodies to human immunodeficiency virus (HIV)-1 and are believed to be an impediment to vaccine development ${ }^{26}$. To determine whether a

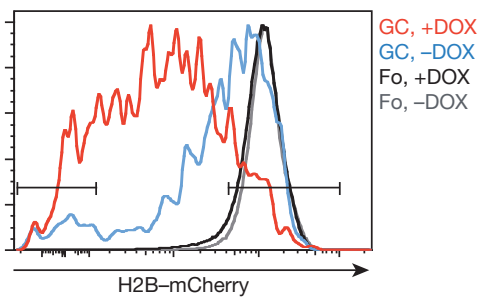

b

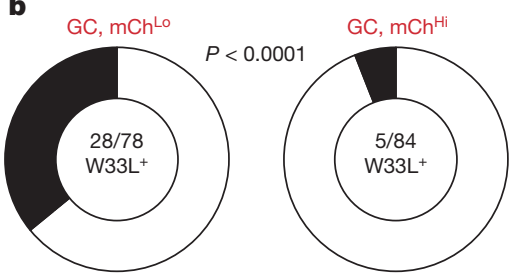

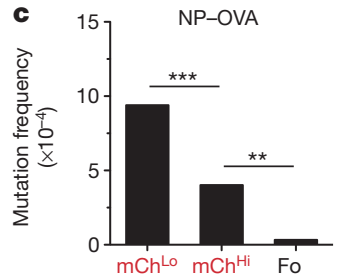

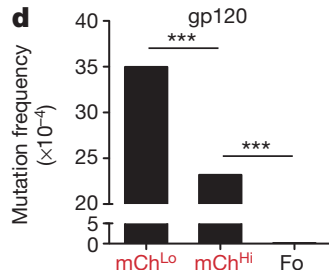

Figure $4 \mid$ Increased cell division in polyclonal GCs is associated with higher immunoglobulin affinity and somatic hypermutation. a-d, tTA-H2B-mCh mice were immunized with NP-OVA $(\mathbf{a}-\mathbf{c})$ or YU2-gp120 (d) and administered DOX for $36 \mathrm{~h}$ before purification. a, Representative histogram displaying $\mathrm{mCh}^{\mathrm{Hi}}$ and $\mathrm{mCh}^{\mathrm{Lo}}$ gates. b. Frequency of W33L mutation among $\mathrm{V}_{\mathrm{H}} 186.2$ clones in $\mathrm{mCh}^{\mathrm{Hi}}$ and $\mathrm{mCh}^{\mathrm{Lo}} \mathrm{GC} \mathrm{B}$ cells on day 14 after immunization. The number of $\mathrm{W}_{33} \mathrm{~L}^{+}$clones among $\mathrm{V}_{\mathrm{H}} 186.2$ sequences is shown in the centre of the pie chart and was compared using Fisher's exact test. Data are pooled from 2 independent experiments with 2 mice each. $\mathbf{c}, \mathbf{d}$, Somatic hypermutation in the $J_{H} 4$ intron among $\mathrm{mCh}^{\mathrm{Hi}}$ and $\mathrm{mCh}^{\mathrm{Lo}} \mathrm{GC} \mathrm{B}$ cells on day 7 after NP-OVA (c) and day 9 after immunization with HIV-1 $1_{\mathrm{YU} 2} \operatorname{gp} 120$ (d). Data in $\mathbf{c}$ and $\mathbf{d}$ are pooled from 2 independent experiments with 2-3 mice each. Over 160 clones in $\mathbf{c}$ and 140 clones in d were analysed for $\mathrm{mCh}^{\mathrm{Hi}}$ and $\mathrm{mCh}^{\mathrm{Lo}} \mathrm{GC} \mathrm{B}$ cells and 51 clones in $\mathbf{c}$ and 94 clones in $\mathbf{d}$ were analysed for follicular B cells. Fo, follicular B cells. ${ }^{* *} P=0.0033 ; * * * P<0.0001, \chi^{2}$ test with Yates correction.

mutations accumulate differentially among GC B cells responding to HIV-1 gp120, we immunized tTA-H2B-mCh mice with HIV-1 1 Y2 gp120 and compared GC B cells that had undergone different levels of division. Similar to NP-OVA, the more divided $\mathrm{mCh}^{\mathrm{Lo}} \mathrm{B}$ cells in the anti-gp120 GCs were significantly more mutated than either $\mathrm{mCh}^{\mathrm{Hi}}$ GC or follicular B cells (Fig. 4d). We conclude that B cells undergo a variable number of divisions in the DZ before returning to the $\mathrm{LZ}$, and that increased cell division is associated with higher immunoglobulin affinity and increased somatic hypermutation.

The GC is a site of intense immunoglobulin diversification and selection, from which high-affinity B cells emerge that seed the memory and plasma cell compartments ${ }^{3,4,27}$. Affinity-based immunoglobulin selection is accomplished iteratively. B-cell clones expanding and mutating in the DZ travel to the LZ to compete for help from $\mathrm{T}_{\mathrm{FH}}$ cells, with only a fraction of LZ cells selected to return to the DZ to continue cycling ${ }^{10}$. Our experiments indicate that the number of GC B-cell divisions per $\mathrm{DZ}$ cycle is variable, ranging from 1 to 6 , and is regulated by the amount of antigen captured in the LZ. By capturing more antigen and dividing a greater number of times in the DZ during each interzonal cycle, highaffinity GC B cells outcompete lower affinity cells that capture less antigen and divide fewer times. The magnitude of T-cell help provided in the LZ therefore regulates the behaviour of a DZ cell-inducing selected cells to re-enter the cell cycle a variable number of times before migrating back to the LZ. This finding is consistent with and may explain the observation that the switch from DZ to LZ phenotype is independent of cues received in the $\mathrm{DZ}^{14}$. Moreover, the least proliferative GC B cells have the fewest affinity-enhancing mutations and are disproportionately found in the LZ (Figs $2 \mathrm{~d}$ and $4 \mathrm{~b}$ ). The precise contributions of death and differentiation to the eventual disappearance of these cells from the GC remain to be determined.

An important feature of differential cell division in the dark zone is that each round of division is associated with increased accumulation of somatic hypermutation. Consistent with this idea, highly proliferative germinal centre B cells have both higher immunoglobulin affinity and 
a greater number of somatic mutations. Our experiments reveal a feedforward loop in the germinal centre, whereby somatic hypermutation is greatest among germinal centre $B$ cells whose antibodies provide the highest affinity template for further diversification. This finding may be particularly relevant for designing immunization strategies that elicit broadly neutralizing antibodies to pathogens, like HIV-1, that require exceptionally high levels of mutation.

\section{METHODS SUMMARY}

Vav-tTA transgenic mice were bred with Col1A1-tetO-H2B-mCherry mice to produce tTA-H2B-mCh mice ${ }^{21,22}$. B1- $8^{\text {hi }}$, DEC205 ${ }^{-1-}$ and PAGFP transgenic mice were described previously ${ }^{10,19,28}$. Resting $\mathrm{B}$ cells from indicated mice were purified by magnetic cells sorting (MACS) and transferred intravenously into recipient mice that were primed 2-6 weeks previously by intraperitoneal immunization with $50 \mu \mathrm{g}$ of OVA in alum. Adoptive transfer recipients were boosted subcutaneously with $25 \mu \mathrm{g}$ of $\mathrm{NP}_{14}-\mathrm{OVA}$. $\alpha \mathrm{DEC}-\mathrm{OVA}$ and $\alpha \mathrm{DEC}-\mathrm{CS}$ chimaeric antibodies were produced by transient transfection of 293T cells and were injected subcutaneously as indicated. Polyclonal GCs were generated by immunizing tTA-H2B-mCh mice intraperitoneally and subcutaneously with $50 \mu \mathrm{g}$ and $12.5 \mu \mathrm{g}$ of $\mathrm{NP}_{14}-\mathrm{OVA}$ or $12.5 \mu \mathrm{g}$ and $6.25 \mu \mathrm{g}$ of $\mathrm{HIV}-1_{\mathrm{YU} 2}$ gp120 protein in alum, respectively. DOX was administered by injecting $1.6 \mathrm{mg}$ intraperitoneally and $0.2 \mathrm{mg}$ subcutaneously and by supplementing the drinking water with $\operatorname{DOX}\left(2 \mathrm{mg} \mathrm{ml}^{-1}\right)$ and sucrose $\left(10 \mathrm{mg} \mathrm{ml}^{-1}\right)$. Intravital imaging and photoactivation were performed as described previously $\mathrm{y}^{10,18,29}$ Immunoglobulin sequence analysis was performed using MacVector 12.7 and IMGT/ V-QUEST.

Online Content Any additional Methods, Extended Data display items and Source Data are available in the online version of the paper; references unique to these sections appear only in the online paper.

Received 17 January; accepted 1 April 2014.

Published online 4 May 2014.

1. Berek, C., Berger, A. \& Apel, M. Maturation of the immune response in germinal centers. Cell 67, 1121-1129 (1991).

2. Jacob, J., Kelsoe, G., Rajewsky, K. \& Weiss, U. Intraclonal generation of antibody mutants in germinal centres. Nature 354, 389-392 (1991).

3. Rajewsky, K. Clonal selection and learning in the antibody system. Nature $\mathbf{3 8 1}$ 751-758 (1996).

4. Victora, G. D. \& Nussenzweig, M. C. Germinal centers. Annu. Rev. Immunol. 30, 429-457 (2012)

5. Allen, C. D., Okada, T., Tang, H. L. \& Cyster, J. G. Imaging of germinal center selection events during affinity maturation. Science 315, 528-531 (2007).

6. Hauser, A. E. et al. Definition of germinal-center $B$ cell migration in vivo reveals predominant intrazonal circulation patterns. Immunity 26, 655-667 (2007)

7. Kocks, C. \& Rajewsky, K. Stepwise intraclonal maturation of antibody affinity through somatic hypermutation. Proc. Natl Acad. Sci. USA 85, 8206-8210 (1988).

8. McKean, D. et al. Generation of antibody diversity in the immune response of BALB/c mice to influenza virus hemagglutinin. Proc. Natl Acad. Sci. USA 81, 3180-3184 (1984)

9. Schwickert, T. A. et al. In vivo imaging of germinal centres reveals a dynamic open structure. Nature 446, 83-87 (2007)

10. Victora, G. D. etal. Germinal center dynamics revealed by multiphoton microscopy with a photoactivatable fluorescent reporter. Cell 143, 592-605 (2010).

11. Allen, C. D., Okada, T. \& Cyster, J. G. Germinal-center organization and cellular dynamics. Immunity 27, 190-202 (2007).

12. Oprea, M. \& Perelson, A. S. Somatic mutation leads to efficient affinity maturation when centrocytes recycle back to centroblasts. J. Immunol. 158, 5155-5162 (1997).
13. Allen, C. D. et al. Germinal center dark and light zone organization is mediated by CXCR4 and CXCR5. Nature Immunol. 5, 943-952 (2004).

14. Bannard, O. et al. Germinal center centroblasts transition to a centrocyte phenotype according to a timed program and depend on the dark zone for effective selection. Immunity 39, 912-924 (2013).

15. Dominguez-Sola, D. et al. The proto-oncogene MYC is required for selection in the germinal center and cyclic reentry. Nature Immunol. 13, 1083-1091 (2012).

16. Jiang, W. et al. The receptor DEC-205 expressed by dendritic cells and thymic epithelial cells is involved in antigen processing. Nature 375, 151-155 (1995).

17. Kamphorst, A. O., Guermonprez, P., Dudziak, D. \& Nussenzweig, M. C. Route of antigen uptake differentially impacts presentation by dendritic cells and activated monocytes. J. Immunol. 185, 3426-3435 (2010).

18. Shulman, Z. et al. T follicular helper cell dynamics in germinal centers. Science 341, 673-677 (2013).

19. Shih, T. A., Roederer, M. \& Nussenzweig, M. C. Role of antigen receptor affinity in T cell-independent antibody responses in vivo. Nature Immunol. 3, 399-406 (2002).

20. Boscardin, S. B. etal. Antigen targeting to dendritic cells elicits long-lived T cell help for antibody responses. J. Exp. Med. 203, 599-606 (2006).

21. Egli, D., Rosains, J., Birkhoff, G. \& Eggan, K. Developmental reprogramming after chromosome transfer into mitotic mouse zygotes. Nature 447, 679-685 (2007).

22. Wiesner, S. M., Jones, J. M., Hasz, D. E. \& Largaespada, D. A. Repressible transgenic model of NRAS oncogene-driven mast cell disease in the mouse. Blood 106 1054-1062 (2005)

23. Tumbar, T. et al. Defining the epithelial stem cell niche in skin. Science $\mathbf{3 0 3}$, 359-363 (2004)

24. Allen, D., Simon, T., Sablitzky, F., Rajewsky, K. \& Cumano, A. Antibody engineering for the analysis of affinity maturation of an anti-hapten response. EMBO J. 7, 1995-2001 (1988)

25. Jolly, C. J., Klix, N. \& Neuberger, M. S. Rapid methods for the analysis of immunoglobulin gene hypermutation: application to transgenic and gene targeted mice. Nucleic Acids Res. 25, 1913-1919 (1997).

26. West, A. P., Jretal. Structural insights on the role of antibodies in HIV-1 vaccine and therapy. Cell 156, 633-648 (2014).

27. Tarlinton, D. \& Good-Jacobson, K. Diversity among memory B cells: origin, consequences, and utility. Science 341, 1205-1211 (2013).

28. Guo, M. et al. A monoclonal antibody to the DEC-205 endocytosis receptor on human dendritic cells. Hum. Immunol. 61, 729-738 (2000).

29. Schwickert, T. A. et al. A dynamic T cell-limited checkpoint regulates affinity-dependent B cell entry into the germinal center. J. Exp. Med. 208, 1243-1252 (2011).

Acknowledgements We thank S. W. Lowe and D. R. Fooksman for mice; D. Bosque and T. Eisenreich for help with mouse colony management; A. Abadir for protein production; K. Yao for technical help; K. Velinzon for help with cell sorting; D. Mucida and all members of the Nussenzweig laboratory for discussion. Support for the Rockefeller University multiphoton microscope was granted by the Empire State Stem Cell Fund through New York State Department of Health contract C023046. Supported by National Institutes of Health (NIH) Medical Scientist Training Program grant T32GM07739 to the Weill Cornell/Rockefeller/Sloan-Kettering Tri-Institutional MD-PhD Program (A.D.G.); NIH grants Al037526-19 and Al072529-06 (M.C.N.); and the NIH Center for HIV/AIDS Vaccine Immunology and Immunogen Discovery (CHAVI-ID) 1UM1 Al100663-01 (M.C.N). Z.S. is a Human Frontiers of Science Fellow. M.C.N. is an HHMI investigator.

Author Contributions A.D.G. planned and performed experiments and wrote the manuscript. A.D.G. and Z.S. planned and performed photoactivation experiments. M.C.N. planned experiments and wrote the manuscript.

Author Information Reprints and permissions information is available at www.nature.com/reprints. The authors declare no competing financial interests. Readers are welcome to comment on the online version of the paper. Correspondence and requests for materials should be addressed to M.C.N. (nussen@mail.rockefeller.edu). 


\section{METHODS}

Mice. C57/BL6, B6.SJL, and Col1A1-tetO-H2B-mCherry mice were purchased from Jackson Laboratories. Vav-tTA transgenic mice that had been backcrossed to C57/BL6 were obtained from S. W. Lowe and crossed to Col1A1-tetO-H2B-mCherry mice to produce tTA-H2B-mCh mice ${ }^{21,22} . \mathrm{B} 1-8^{\mathrm{hi}}, \mathrm{DEC} 205^{-1-}$, and PAGFP transgenic mice were described previously ${ }^{10,19,28}$. All experiments were performed with authorization from the Institutional Review Board and the IACUC at The Rockefeller University.

B-cell transfer and culture. Resting B cells were purified by forcing spleen tissue through a $40-\mu \mathrm{m}$ mesh into complete RPMI media (Gibco) with $6 \%$ serum. Singlecell suspensions were purified by magnetic cells sorting (MACS) using CD43 beads, according to the manufacturer's instructions (Miltenyi Biotec). Indicated cell numbers were transferred intravenously into recipient mice. To assess proliferation in vitro, resting $\mathrm{B}$ cells purified from $\mathrm{tTA}-\mathrm{H} 2 \mathrm{~B}-\mathrm{mCh}$ mice were labelled at $37^{\circ} \mathrm{C}$ in $5 \mu \mathrm{M}$ carboxyfluorescein succinimidyl ester. Labelled B cells were then stimulated with lipopolysaccharide and IL- 4 for $72 \mathrm{~h}$ as previously described ${ }^{30}$. DOX was added to the cultures at $500 \mathrm{ng} \mathrm{ml}^{-1}$.

Immunizations and treatments. C57BL/6 or B6.SJL male recipient mice (6-8 weeks of age) were primed by intraperitoneal immunization with $100 \mu \mathrm{l}$ containing $50 \mu$ g of OVA (Grade V, Sigma) precipitated in alum at a 2:1 ratio in PBS. Two to six weeks after priming, mice received adoptive cell transfers of indicated $\mathrm{B}$ cells and were boosted the following day with $25 \mu \mathrm{g}$ of $\mathrm{NP}_{14}$-OVA (Biosearch Technologies) in hind footpads. Popliteal lymph nodes were collected for flow cytometric analysis. To generate polyclonal GCs, mice were immunized with $50 \mu \mathrm{g}$ intraperitoneally and $12.5 \mu \mathrm{g}$ in the hind footpads of $\mathrm{NP}_{14}-\mathrm{OVA}$ in alum. HIV-1 $1_{\mathrm{YU}}$ gp120specific GCs were induced by immunizing mice intraperitoneally and in hind footpads with $12.5 \mu \mathrm{g}$ and $6.25 \mu \mathrm{g}$ of gp120 protein, respectively, in alum. $\alpha \mathrm{DEC}-$ OVA and $\alpha$ DEC-CS chimaeric antibodies were produced by transient transfection in $293 \mathrm{~T}$ cells, as described ${ }^{20} .10 \mu \mathrm{g}$ of chimaeric antibody in PBS was injected into footpads of mice at indicated time points, except in Fig. 3b in which $5 \mu \mathrm{g}$ of chimaeric antibody was injected into footpads. Mice were administered DOX by intraperitoneal injection of $1.6 \mathrm{mg}$ DOX (Sigma) in PBS and hind footpad injection of $0.2 \mathrm{mg}$ DOX in PBS. Mice were maintained on DOX by adding DOX $\left(2 \mathrm{mg} \mathrm{ml}^{-1}\right)$ and sucrose $\left(10 \mathrm{mg} \mathrm{ml}^{-1}\right)$ to the drinking water for the indicated periods of time. Flow cytometry. Lymph nodes were collected by forcing tissue through $40-\mu \mathrm{m}$ mesh into complete RPMI media (Gibco) with $6 \%$ serum. Single cell suspensions were treated at $4{ }^{\circ} \mathrm{C}$ for $10 \mathrm{~min}$ with $1 \mu \mathrm{g} \mathrm{ml}^{-1}$ anti-CD16/32 (2.4G2, Bio-X-Cell) and then stained for $25 \mathrm{~min}$ at $4{ }^{\circ} \mathrm{C}$. Anti-B220, CD38, CD86, CD45.1 and CD45.2 antibodies were from eBioscience. FAS, CXCR4, CD45.1, CD45.2, Ig $\lambda_{1-3}$, GL7, streptavidinphycoerythrin and streptavidin-allophycocyanin were from BD Biosciences. StreptavidinAlexa Fluor 488 was from Invitrogen. For cell cycle and S phase analysis, mice were injected intravenously with $2 \mathrm{mg} \mathrm{BrdU}$ (Sigma-Aldrich) and $1 \mathrm{mg}$ EdU (Life Technologies) in PBS. Cells were then stained for surface antigens as described above and processed using an anti-BrdU-FITC kit (BD Biosciences) and Click-iT EdUPacific Blue kit (Life Technologies) according to manufacturers' protocols. All samples were analysed on a BD Fortessa. GC B cells were gated as live/single, B220 ${ }^{+}$ $\mathrm{CD}^{-} 8^{-}$and $\mathrm{FAS}^{+}$. DZ and LZ GC B cells were further gated as CXCR $4^{+} \mathrm{CD}^{-} 6^{-}$ and $\mathrm{CXCR} 4^{-} \mathrm{CD} 6^{+}$, respectively. $\mathrm{CD} 45.1$ and $\mathrm{CD} 45.2$ allotypic markers were used to trace adoptively transferred B cells of genotypes B1- $8^{\text {hi }} \mathrm{DEC} 205^{+/+}\left(\mathrm{CD} 45.1^{+}\right)$, B1- $8^{\text {hi }}$ DEC205 $5^{-l-}\left(\mathrm{CD} 45.1^{+} \mathrm{CD} 45.2^{+}\right)$, and B1- $8^{\text {hi }}$ DEC205 $5^{+/+}$tTA-H2B-mCh $\left(\mathrm{CD} 45.2^{+}\right)$within either C57/BL6 $\left(\mathrm{CD} 45.2^{+}\right)$or B6.SJL $\left(\mathrm{CD} 45.1^{+}\right)$recipient mice. Photoactivation. Intravital imaging and photoactivation were performed as described previously ${ }^{10,18,29}$. Anaesthesia was induced with $100 \mathrm{mg}$ ketamine, $15 \mathrm{mg}$ xylazine and $2.5 \mathrm{mg}$ acepromazine per $\mathrm{kg}$ of body weight and maintained with $1.25 \%$ isoflurane in $100 \%$ oxygen. A double-edged razor blade was used to shave hind legs. Mice were restrained on a $37^{\circ} \mathrm{C}$ stage warmer (BioTherm Micro S37; Biogenics) and an incision was made behind the knee to allow exposure of the popliteal lymph node. Once exposed, the lymph node was restrained with a metal strap and visualized with a microscope objective and a $40^{\circ} \mathrm{C}$ objective heater. To label LZ-resident follicular dendritic cells, $1 \mu \mathrm{g}$ of the red fluorescent protein tdTomato conjugated to NP was injected in hind footpads one day before imaging ${ }^{10,18}$. To photoactivate DZ B cells, B1-8 ${ }^{\text {hi }}$ PAGFP $^{+}$GC B cells were photoactivated external to NP-tdTomatolabelled FDCs in the LZ by scanning with a femtosecond-pulsed multiphoton laser tuned to $820 \mathrm{~nm}$ wavelength and imaged at $940 \mathrm{~nm}$ wavelength, as previously described ${ }^{10,18}$. Cell motility was monitored immediately following photoactivation to ensure cell viability. Incisions were sutured and mice were allowed to recover for $6 \mathrm{~h}$ before flow cytometric analysis. To determine the accuracy of this strategy, B1- $8^{\text {hi }}$ PAGFP $^{+}$GC B cells were photoactivated in the DZ of explanted lymph nodes and analysed immediately for surface phenotype. Imaging experiments were performed with an Olympus BX61 upright microscope (Olympus $\times 251.05 \mathrm{NA}$ Plan objective), fitted with a Coherent Chameleon Vision II laser (Rockefeller University Bio-Imaging Resource Center).

Immunoglobulin sequence analysis. B cells were purified from spleen and lymph node tissues of immunized mice using CD43 magnetic beads (MACS). Single cell suspensions were stained as described above and sorted using a FACS Aria II (Becton Dickinson). GC B cells were gated as live/single, B220 ${ }^{+} \mathrm{CD} 38^{-} \mathrm{FAS}^{+}$(Fig. 4c) or B220 ${ }^{+} \mathrm{CD}_{3} 8^{-} \mathrm{FAS}^{+} \mathrm{GL}^{+}$(Fig. $4 \mathrm{~d}$ ) and $\mathrm{mCh}^{\mathrm{Hi}}$ or $\mathrm{mCh}^{\mathrm{Lo}}$. Genomic DNA was extracted from sorted cell populations and PCR was performed from DNA corresponding to 1,000-5,000 cells using Phusion HF (New England Biolabs). PCR products were gel extracted and cloned into Zero Blunt TOPO vectors (Invitrogen). For $V_{H} 186.2$ sequence analysis, GC B cells were further gated as $\operatorname{Ig} \lambda^{+}$and clones were amplified as described previously $y^{10,15}$. Immunoglobulin sequences were analysed using the IMGT/V-QUEST system to identify W33L mutations. $J_{H} 4$ intronic sequences were amplified using $5^{\prime}$-TCCTAGGAACCAACTTAAGAGT- ${ }^{\prime}$ and $5^{\prime}$-TGGAGTTT TCTGAGCATTGCAG- $3^{\prime}$ primers and 35 cycles with an annealing temperature of $57^{\circ} \mathrm{C}$ and an extension time at $72^{\circ} \mathrm{C}$ for $1 \mathrm{~min}$. High-quality traces were analysed using MacVector 12.7 for base-pair mismatches and deletions as compared to the germline sequence. Both mismatches and deletions were counted as mutations. To calculate mutation frequency, the total number of mutations from all clones was summed and divided by the total number of base pairs analysed for each group. Mutation frequencies among different groups were compared and analysed for statistical significance by the $\chi^{2}$ test with Yates correction using Prism software v. 5.0 (Graphpad).

30. Robbiani, D. F. et al. AID is required for the chromosomal breaks in c-myc that lead to c-myc/lgH translocations. Cell 135, 1028-1038 (2008). 
a live/single, B220+:

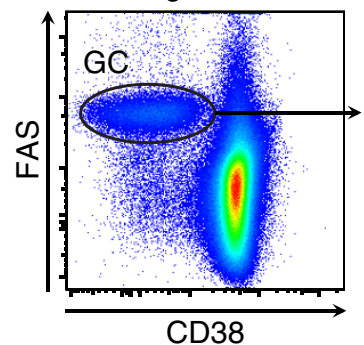

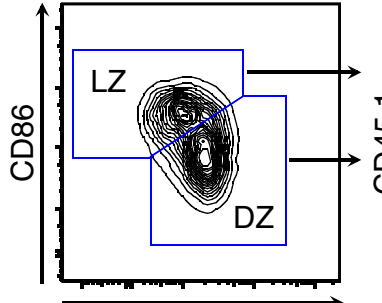

CXCR4

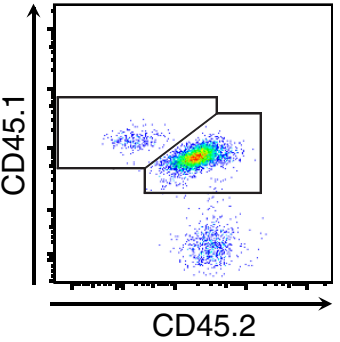

CD45.2

b

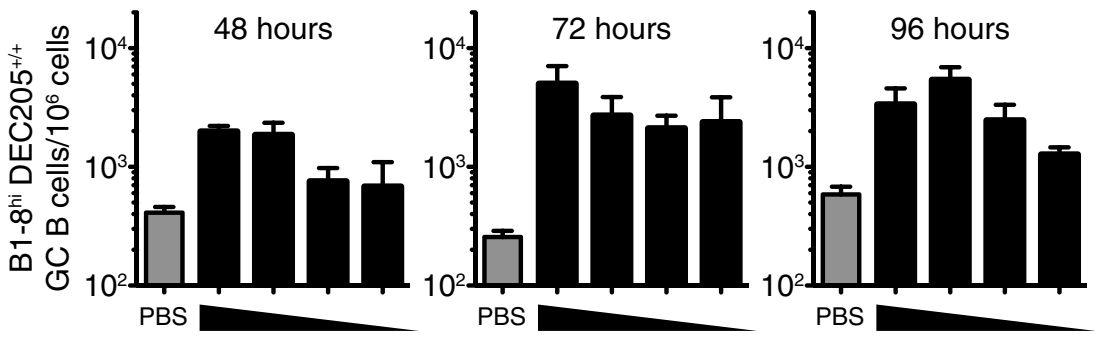

C

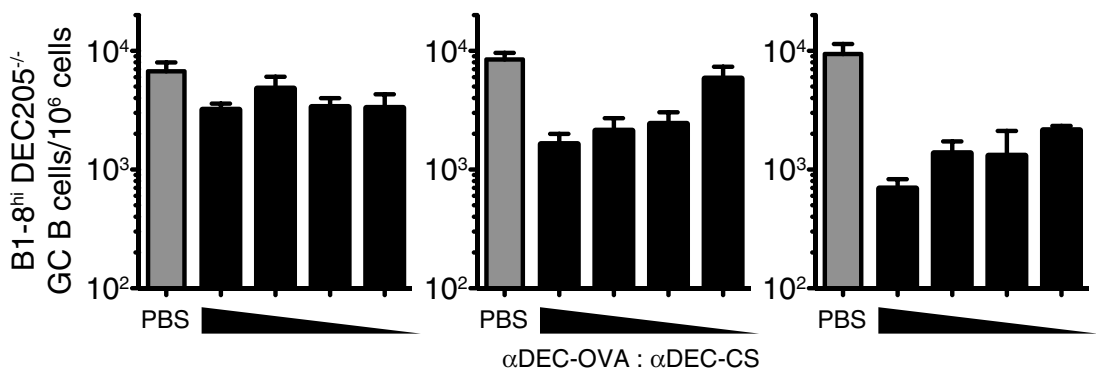

Extended Data Figure $1 \mid$ Flow cytometric analysis of germinal centre B cells. a, Representative flow cytometry plots display gating strategy used to analyse experiments in Fig. 1. Live $\mathrm{B}_{22} 20^{+}$singlets were gated on GC cells (CD38-FAS+) and divided into $\mathrm{LZ}\left(\mathrm{CD} 86^{+} \mathrm{CXCR}^{-}\right)$and $\mathrm{DZ}$ $\left(\mathrm{CD} 86^{-} \mathrm{CXCR}^{+}\right)$cells. B1-8 ${ }^{\text {hi }}$ DEC205 ${ }^{+/+}$cells within these compartments were identified as CD45. $1^{+}$and B1-8 $8^{\text {hi }} \mathrm{DEC} 205^{-1-}$ cells were identified as CD $45.1^{+}$CD $45.2^{+} . \mathbf{b}, \mathbf{c}$, Total number of B1- $8^{\text {hi }}$ DEC205 $5^{+/+}(\mathbf{b})$ and B1- $8^{\text {hi }}$ DEC205 $5^{-1-}$ (c) GC B cells per $10^{6}$ lymph node cells from the experiments reported in Fig. 1. Bars represent mean values; error bars, s.e.m. 


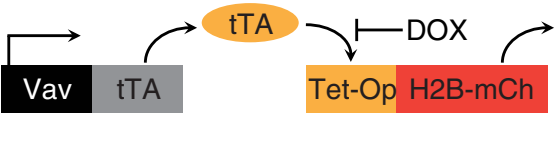

C

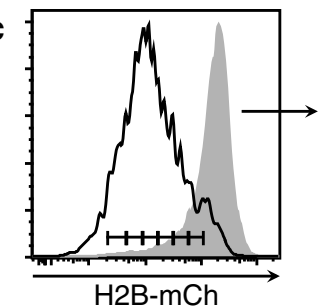

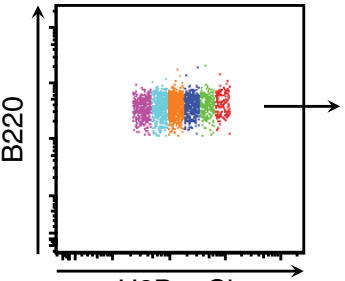

H2B-mCh b

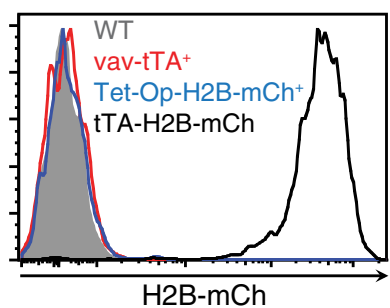

H2B-mCh

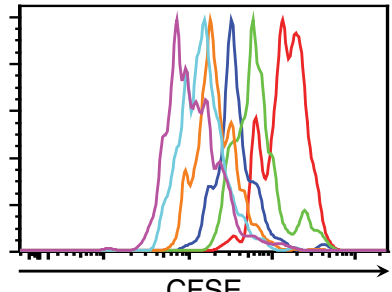

Extended Data Figure $2 \mid$ tTA-H2B-mCh system. a, Diagrammatic representation of the Vav-tTA and Tet-Op-H2B-mCh transgenes that were combined (tTA-H2B-mCh) to label B cells with $\mathrm{H} 2 \mathrm{~B}-\mathrm{mCh}$ in order to inducibly measure cell division in the GC with DOX. b. Histogram displaying $\mathrm{H} 2 \mathrm{~B}-\mathrm{mCh}$ expression among B220 ${ }^{+}$lymphocytes from the peripheral blood of wild-type (grey), Vav-tTA ${ }^{+}$(red), Tet-Op-H2B-mCh ${ }^{+}$(blue), and tTA-H2B-mCh mice (black). c, Purified B cells from tTA-H2B-mCh mice were labelled with carboxyfluorescein succinimidyl ester (CFSE) and stimulated with lipopolysaccharide and IL-4. Left, H2B-mCh levels after 0 or $72 \mathrm{~h}$ in culture (grey and black, respectively). Middle, $\mathrm{H} 2 \mathrm{~B}-\mathrm{mCh}$ gates for $\mathrm{B}$ cells activated for $72 \mathrm{~h}$ are colour-coded. Right, histogram displaying the CFSE levels for the colour-coded $\mathrm{H} 2 \mathrm{~B}-\mathrm{mCh}$ gates. Data are representative of two independent experiments. 
a

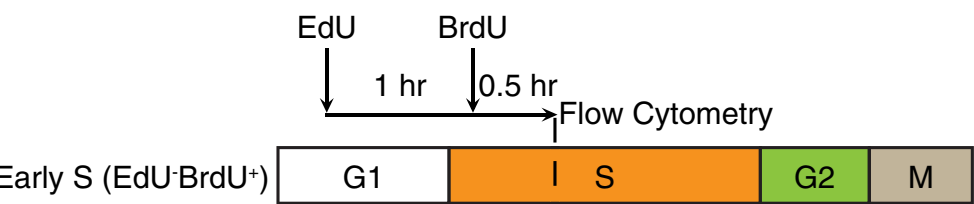

$\mathrm{Mid} /$ late S (EdU $\left.{ }^{+B r d U}{ }^{+}\right)$

\begin{tabular}{|l|l|l|l|l|}
\hline G1 & S & I 2 & M \\
\hline
\end{tabular}

\begin{tabular}{|c|c|c|c|c|}
\hline Post-S (EdU'BrdU') & G1 & $\mathrm{S}$ & G2 & $M$ \\
\hline
\end{tabular}
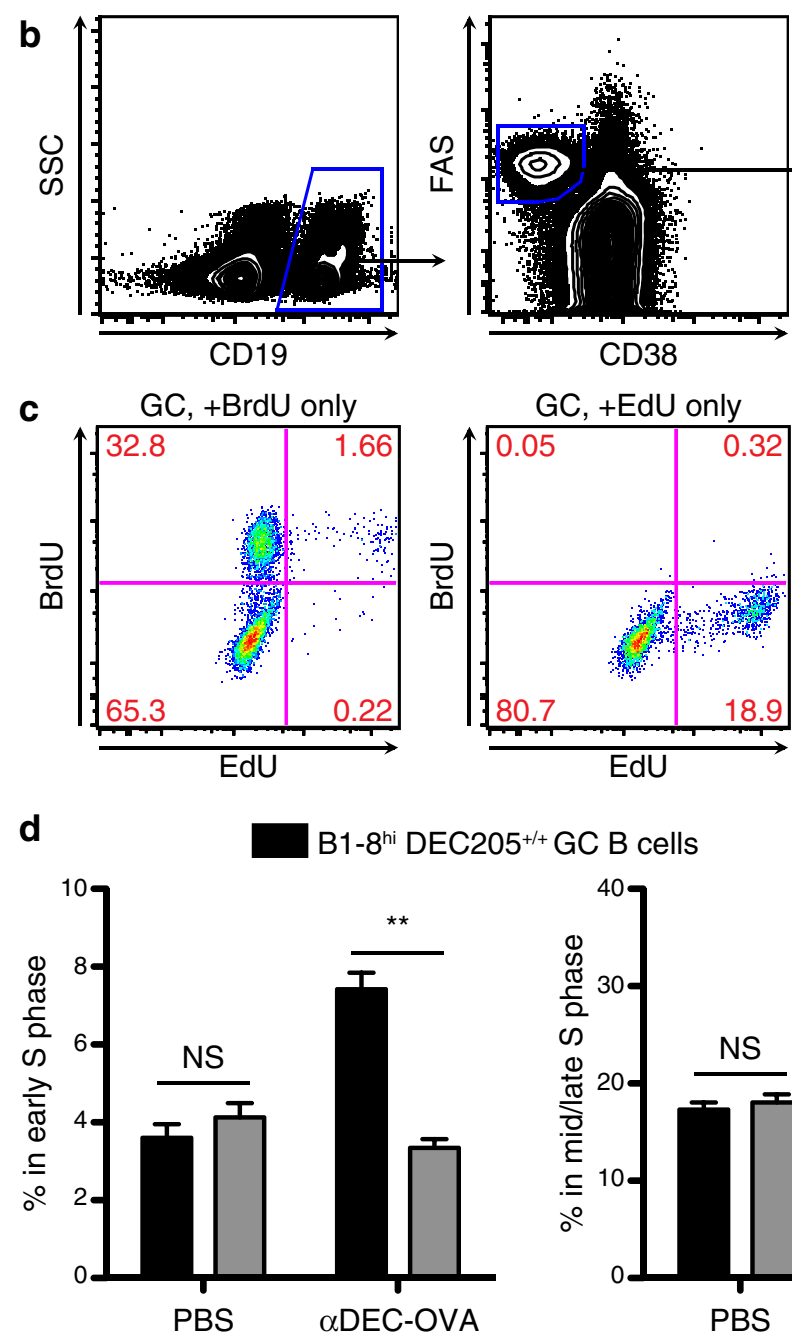

Extended Data Figure $3 \mid$ Edu/BrdU labelling strategy to analyse the progression of S phase. a, Mice with ongoing GCs were administered intravenous EdU followed by intravenous BrdU $1 \mathrm{~h}$ later. A half-hour after BrdU administration, mice were analysed by flow cytometry. Cells in early $S$ phase at the time of analysis incorporate only the second nucleotide analogue and can therefore be identified as $\mathrm{EdU}^{-} \mathrm{BrdU}^{+}$. Cells in mid/late $\mathrm{S}$ phase replicate DNA during both the EdU and BrdU injections and are therefore $\mathrm{EdU}^{+} \mathrm{BrdU}^{+}$. Cells that completed $S$ phase in the hour between EdU and BrdU administration are post-S phase cells at the time of analysis. These cells incorporate the first label, but not the second, making them $\mathrm{EdU}^{+} \mathrm{BrdU}^{-}$. b, Gating strategy used in Fig. 3a, b. B1- $8^{\text {hi }}$ DEC205 $5^{+/+}$and B1- $8^{\text {hi }}$ DEC205 $5^{-/-}$
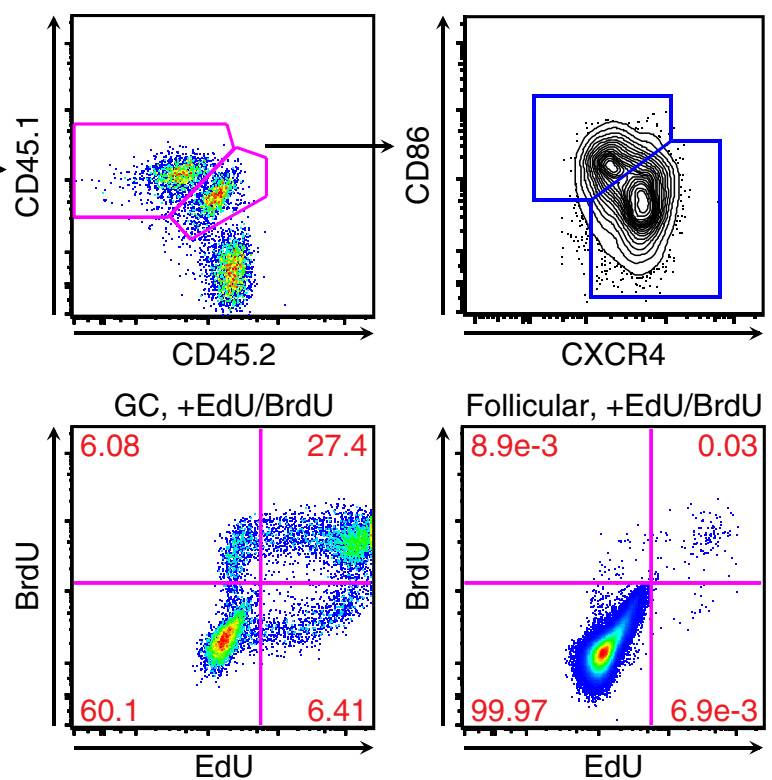

Follicular, +EdU/BrdU

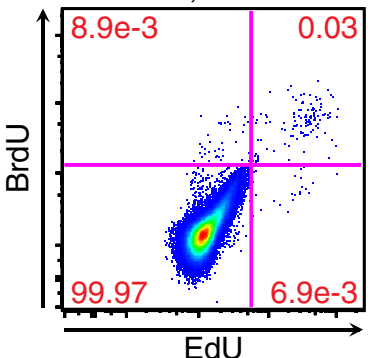

B1-8 ${ }^{\text {hi }}$ DEC205\%- GC B cells
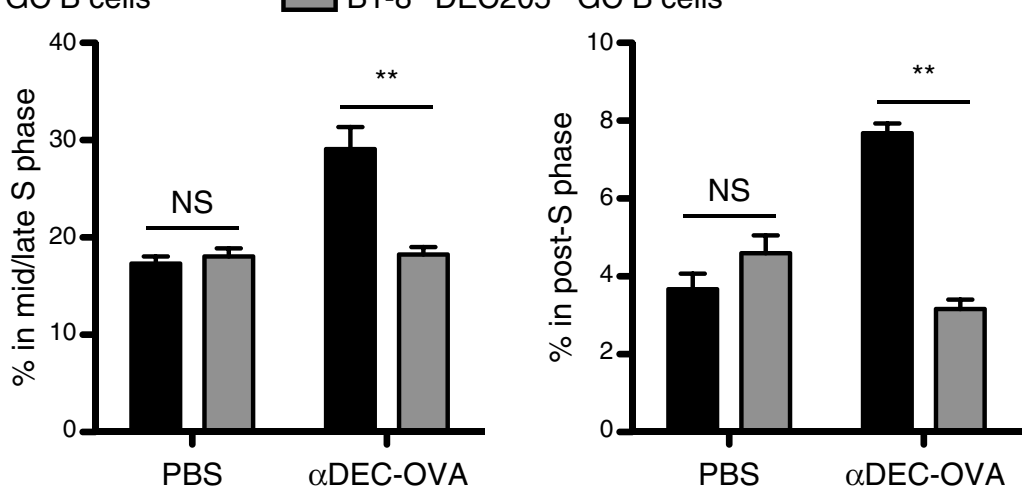

GC B cells were identified among $\mathrm{CD} 19^{+} \mathrm{CD} 38^{-} \mathrm{FAS}^{+}$cells using CD45 allotypic markers and were further subdivided based on EdU and BrdU incorporation and DZ/LZ surface phenotype. c, Flow cytometry plots displaying EdU and BrdU incorporation in GC and follicular B cells of mice receiving EdU and/or BrdU. d, Per cent of B1- $8^{\text {hi }}$ DEC205 $5^{+/+}$(black) and B1-8 ${ }^{\text {hi }}$ DEC205 ${ }^{-1-}$ (grey) GC B cells in early ( $\mathrm{EdU}^{-} \mathrm{BrdU}^{+}$, left), mid/late $\left(\mathrm{EdU}^{+} \mathrm{BrdU}^{+}\right.$, middle), and post- $\left(\mathrm{EdU}^{+} \mathrm{BrdU}^{-}\right.$, right $) \mathrm{S}$ phase periods in control (PBS) or $\alpha$ DEC-OVA treated mice. Data represent values from the same experiments reported in Fig. 3a, b. Error bars, s.e.m; ** $P=0.0022$, two-tailed Mann-Whitney test. 
a

B cell transfer:

$15 \%$ B1-8 ${ }^{\text {hi }}$ DEC205 $5^{+/+}$PAGFP+

$85 \%$ B $1-8^{\text {hi }}$ DEC205 ${ }^{-/}$

OVA/alum i.p. $\quad 100 \%{\mathrm{~B} 1-8^{\text {hi }} \text { DEC205 }}^{+/+}$PAGFP $^{+}$
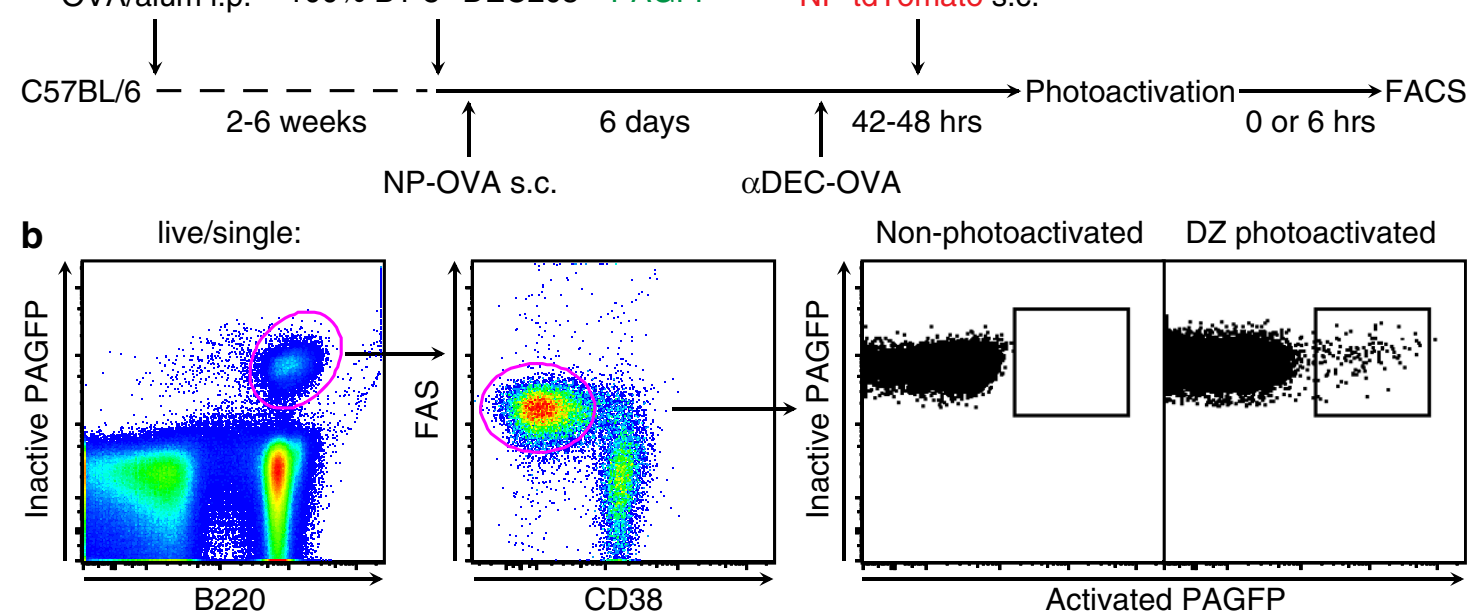

-OVA S.c.

$\alpha$ DEC-OVA
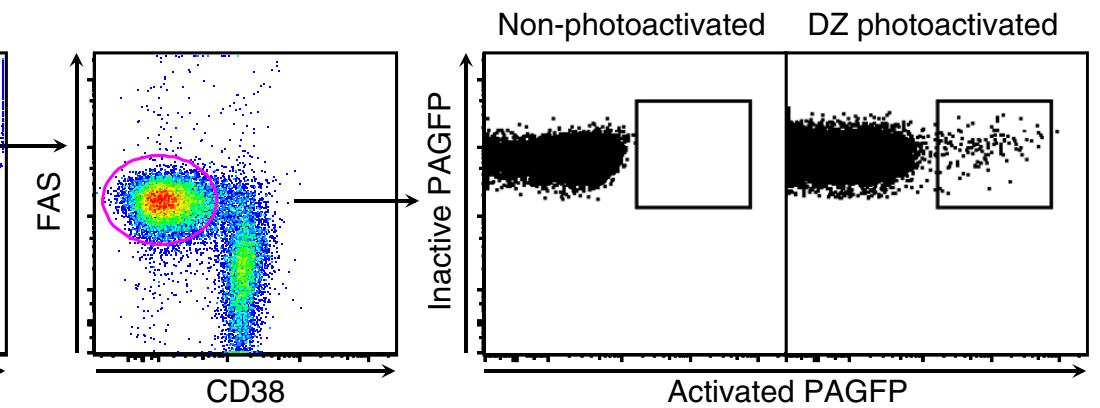

Extended Data Figure $4 \mid \mathrm{DZ}$ photoactivation protocol and flow cytometric analysis. a, Diagrammatic representation of the protocol used in Fig. 3c-e.

the DZ. Live singlets were gated as $\mathrm{B}_{22}{ }^{+} \mathrm{PAGFP}^{+} \mathrm{CD} 38^{-} \mathrm{FAS}^{+}$Active b, Flow cytometric gating strategy used to analyse GC B cells photoactivated in the DZ. Live
PAGFP $^{+}$. 